\title{
The Correlation between FSTL1 Expression and Airway Remodeling in Asthmatics
}

\author{
Yahui Liu, ${ }^{1}$ Tian Liu, ${ }^{1}$ Jinxiang Wu, ${ }^{1}$ Tao Li, ${ }^{2}$ Xingai Jiao, ${ }^{1}$ Haiqing Zhang, ${ }^{2}$ Jiping Zhao, ${ }^{1}$ \\ Junfei Wang, ${ }^{1}$ Lin Liu, ${ }^{1}$ Liuzhao Cao, ${ }^{1}$ Shuo Li, ${ }^{1}$ Jiawei $\mathrm{Xu},{ }^{1}$ Jianfeng Xu, ${ }^{1}$ Xiaohui Ma, \\ Lei Yang, ${ }^{1}$ and Liang Dong ${ }^{1}$ \\ ${ }^{1}$ Department of Pulmonary Medicine, Qilu Hospital of Shandong University, Jinan 250012, China \\ ${ }^{2}$ Department of Pulmonary Medicine-Bronchoscopy Room, Qilu Hospital of Shandong University, Jinan 250012, China
}

Correspondence should be addressed to Liang Dong; dl5506@126.com

Received 4 January 2017; Revised 6 April 2017; Accepted 19 June 2017; Published 3 August 2017

Academic Editor: Sandra Helena Penha Oliveira

Copyright (c) 2017 Yahui Liu et al. This is an open access article distributed under the Creative Commons Attribution License, which permits unrestricted use, distribution, and reproduction in any medium, provided the original work is properly cited.

\begin{abstract}
Background. Asthma is characterized by airway remodeling. Follistatin-like protein 1 (FSTL1) is an extracellular glycoprotein. Recent studies suggest that FSTL1 may participate in the pathogenesis of asthma. Objectives. To analyze the association between FSTL1 and some parameters and inspect the role of FSTL1 in asthma. Methods. We examined FSTL1 levels in 32 asthmatics and 25 controls. All subjects enrolled had routine blood tests, spirometry, and impulse oscillometry performed. Additionally, 15 of the 32 asthmatics underwent fibre optic bronchoscopy. Spearman rank analysis was performed to detect the correlation between FSTL1 and other parameters. Results. Plasma FSTL1 levels were higher in asthmatics $(130.762 \pm 46.029 \mathrm{ng} / \mathrm{mL})$ than in controls $(95.408 \pm 33.938 \mathrm{ng} / \mathrm{mL})(p=0.009)$. Plasma FSTL1 levels were associated with fibrosis levels around the airways ( $\mathrm{rs}=0.529, p=0.043)$ and $\alpha$-smooth muscle actin $(\alpha$-SMA) (rs $=0.554, p=0.032)$. FSTL1 levels in bronchoalveolar lavage fluid were associated with collagen I ( $\mathrm{rs}=0.536, p=0.040), \alpha$-SMA ( $\mathrm{rs}=0.561, p=0.029$ ), fibrosis levels ( $\mathrm{rs}=0.779$, $p=0.001)$, and the thickness of the airway reticular basement membrane $(\mathrm{RBM})(\mathrm{rs}=0.660, p=0.007)$. Conclusions. FSTL1 levels in asthmatics were linked with increased smooth muscle mass and thickened RBM. FSTL1 may contribute to airway remodeling in asthmatics.
\end{abstract}

\section{Introduction}

Asthma is a chronic respiratory disease usually characterized by chronic airway inflammation, airway remodeling, and airway hyper responsiveness [1]. Airway remodeling refers to the structural changes in the airway including, but not limited to, the airway smooth muscle, airway epithelia, blood vessels, and extracellular matrix [2]. Features of airway remodeling in asthma include an increase of airway smooth muscle mass, epithelial injury, epithelial cell hyperplasia, goblet cell metaplasia, reticular basement membrane (RBM) thickening, and angiogenesis [2]. Previous studies have indicated that these structural changes contribute to asthma persistence, airflow obstruction, lung function decline, and clinical severity $[3,4]$. The mechanisms of airway remodeling, however, are still unclear. There is evidence to show that multiple cytokines, chemokines, transcription factors, and growth factors released from inflammatory and structural cells in the airway are involved in airway remodeling, such as transforming growth factor beta (TGF- $\beta$ ), vascular endothelial growth factor (VEGF), Th2 cytokines (IL-5, IL-9, and IL-13), and epithelial-derived nuclear factor kappa-light-chain-enhancer of activated B cells (NF- $\kappa$ B) [5].

Follistatin-like protein 1 (FSTL1), also known as transforming growth factor (TGF) $\beta 1$-stimulated clone 36 (TSC-36) or follistatin-related protein (FRP), is a secreted glycoprotein of 308 amino acids [6, 7]. Although the function of FSTL1 is not completely understood, it has been shown that FSTL1 plays a key role in tumor bone metastasis $[8,9]$, 
chronic pain hypersensitivity [10], inflammation and insulin resistance in obesity [11], the regulation of erythropoiesis [12], physical development [13-16], and somatic sensation by binding to $\mathrm{Na}^{+}, \mathrm{K}^{+}$-ATPase [17]. More importantly, many studies have demonstrated that FSTL1 upregulates proinflammatory mediators in the pathology of arthritis and serum levels of FSTL1 correlating with severity of arthritis [18-20]. However, FSTL1 may offer protection of the heart as it is essential for the acute repair of the infarcted myocardium [21] and can antagonize myocyte hypertrophic growth and the loss of ventricular performance in response to pressure overload [22]. In murine and porcine models of ischemia/reperfusion, FSTL1 prevents myocardial ischemia/ reperfusion injury [23].

Several studies have shown that FSTL1 may play an important role in the respiratory system. FSTL1 modulates lung development, cartilage formation, and alveolar maturation $[13,24,25]$. Knockout of FSTL1 in mice is embryoniclethal, and these mice display multiple developmental abnormalities of the respiratory and skeletal system [13]. Additionally, FSTL1 has a role in cellular proliferation and apoptosis in lung cancer [26]. Lung injury can induce the production of FSTL1 which can promote the accumulation of myofibroblasts and subsequently lead to fibrosis [27]. Miller et al. demonstrated that FSTL1 is highly expressed by macrophages in the lungs of people with severe asthma and that the FSTL1/oncostatin M pathway may promote airway remodeling in severe asthma [28]. Here, we aimed to compare the concentration of FSTL1 in plasma and bronchoalveolar lavage fluid (BALF) in patients with and without asthma. We then aimed to analyze the association between FSTL1 and a series of clinical parameters to help identify mechanisms behind the pathology of airway remodeling in asthma.

\section{Materials and Methods}

2.1. Study Population. In our study, 32 subjects who visited the Respiratory Clinic of Qilu Hospital, Shandong University (Jinan, Shandong, China), and were diagnosed with asthma according to the Global Initiative for Asthma (2015 edition) were enrolled. All patients were required to meet at least one of the following eligibility criteria: a history of wheezing, cough, chest tightness, and/or dyspnea; having a positive bronchodilator response of $12 \%$ and $200 \mathrm{~mL}$ increase in forced expiratory volume in one second (FEV1); and/or day to day airflow variability. All patients were treatment naive prior to enrolment. We also recruited 25 subjects who visited our hospital for a routine medical checkup but did not have any respiratory condition as nonasthmatic controls.

All subjects (asthmatics and controls) had no history of primary cardiomyopathy, secondary cardiomyopathy, congenital heart disease, renal failure, autoimmune diseases, pulmonary fibrosis, cardiac surgery, and coronary artery therapy as these conditions may influence the plasma levels of FSTL1. Patients who had any symptoms of respiratory infection in the 2 weeks prior were also excluded.
The research protocol was approved by the Ethics Review Committee for Human Studies at Qilu Hospital, Shandong University, and all the subjects provided written informed consent.

2.2. Routine Blood and Pulmonary Function Tests. All subjects had routine blood, pulmonary function tests (spirometry), and impulse oscillometry (IOS) at Qilu Hospital of Shandong University. Spirometry was conducted using MasterScreen ${ }^{\mathrm{TM}}$ Pneumo spirometer (Jaeger Co., Hoechberg, Germany) and carried out according to ATS/ERS criteria [29]. IOS was conducted on Jaeger MasterScreen IOS (Jaeger Co.), and tests were performed prior to spirometry. Trials lasted approximately 40 seconds and contained 200-250 separate impulses.

2.3. Bronchoscopy. Flexible bronchoscopy was performed in 15 of the 32 asthmatics, and this operation was approved by local institutional review boards. Written informed consent was obtained from these patients. Postmortem human lungs from donors without history of asthma $(n=5)$ were also obtained from the Qilu Hospital Cadaver Donating Center in a protocol approved by the Ethics Review Committee for Human Studies at Qilu Hospital, Shandong University. Biopsies from these postmortem samples acted as controls.

All bronchoscopies were performed under topical anesthesia using the Olympus BF-UC260F-0L8 bronchofibrevideoscope. Bronchoalveolar lavage (BAL) was performed using 3 aliquots of $1 \mathrm{~mL} / \mathrm{kg} 0.9 \%$ sterile saline (to a maximum of $40 \mathrm{~mL}$ per aliquot) instilled into the right middle lobe or an area of radiographically defined abnormality and the returns pooled. Up to 3 biopsies were taken from each subject. The mean number of biopsies per subject was 1.47 .

2.4. FSTL1 Concentration in Plasma and BALF. FSTL1 levels in plasma and BALF were measured by a commercially available enzyme-linked immunosorbent assay (ELISA) kit from CUSABIO, MD, USA. The kit was used according to the manufacturer's instructions with the standard curve generated using Curve Expert 1.3. The detection range was $12.5 \mathrm{ng} / \mathrm{mL}-800 \mathrm{ng} / \mathrm{mL}$. The intra-assay and interassay coefficients of variation were less than $8 \%$ and $10 \%$, respectively.

2.5. Staining of Bronchial Biopsies and Quantification of Histological Images. Bronchoscopy was performed in 15 of the 32 asthmatics. For normal control samples, postmortem nonasthmatic human lungs $(n=5)$ were obtained from the Qilu Hospital Cadaver Donating Center under a protocol approved by the Ethics Review Committee for Human Studies at Qilu Hospital, Shandong University. And they did not suffer from asthma according to their antemortem history. All specimens were obtained by fine aspiration followed by immersing the samples immediately into a $4 \%$ solution of formaldehyde. After 24 hours, samples were embedded in paraffin and stored at $4^{\circ} \mathrm{C}$ before cutting them into $5 \mu \mathrm{m}$ slices.

Bronchial biopsies and postmortem human lung samples were stained with hematoxylin and eosin, periodic acid silver methenamine, and Sirius red. 
TABLE 1: Patient characteristics.

\begin{tabular}{|c|c|c|c|}
\hline & Controls & Asthmatics & $p$ value \\
\hline$n$ & 25 & 32 & \\
\hline Age (years) & $40.84 \pm 18.665$ & $42.31 \pm 15.422$ & $0.746^{\mathrm{a}}$ \\
\hline Gender, male (\%) & $10(40.000)$ & $11(34.375)$ & $0.662^{\mathrm{b}}$ \\
\hline BMI $\left(\mathrm{kg} / \mathrm{m}^{2}\right)$ & $24.748 \pm 2.976$ & $25.500 \pm 4.537$ & $0.477^{\mathrm{a}}$ \\
\hline WBC\# $\left(\times 10^{9} / \mathrm{L}\right)$ & $6.251 \pm 2.008^{\ddagger}$ & $7.460 \pm 2.787$ & $0.092^{\mathrm{a}}$ \\
\hline neu\# $\left(\times 10^{9} / \mathrm{L}\right)$ & $3.935 \pm 1.998^{\ddagger}$ & $4.609 \pm 2.829$ & $0.348^{\mathrm{a}}$ \\
\hline eos\# $\left(\times 10^{9} / \mathrm{L}\right)$ & $0.100 \pm 0.105^{\ddagger}$ & $0.473 \pm 0.706$ & $0.006^{c}$ \\
\hline neu\% & $61.148 \pm 14.608^{\ddagger}$ & $58.813 \pm 15.927$ & $0.592^{\mathrm{a}}$ \\
\hline eos $\%$ & $1.652 \pm 1.687^{\ddagger}$ & $5.938 \pm 8.378$ & $0.008^{c}$ \\
\hline $\log _{10}($ FSTL1) & $1.945 \pm 0.194$ & $2.091 \pm 0.152$ & $0.002^{\mathrm{a}}$ \\
\hline $\mathrm{FSTL}_{1}(\mathrm{ng} / \mathrm{mL})$ & $95.408 \pm 33.938$ & $130.762 \pm 46.029$ & $0.009^{d}$ \\
\hline $\mathrm{FEV}_{1}$ (\%predicted) & $99.245 \pm 10.236$ & $77.502 \pm 16.989$ & $<0.001^{\mathrm{c}}$ \\
\hline $\mathrm{FEV}_{1} / \mathrm{FVC}$ & $84.787 \pm 3.087$ & $70.261 \pm 10.521$ & $<0.001^{\mathrm{c}}$ \\
\hline $\operatorname{Zrs}(\Omega)$ & $104.252 \pm 27.344$ & $271.991 \pm 91.064$ & $<0.001^{\mathrm{c}}$ \\
\hline Fres $(1 / s)$ & $11.436 \pm 2.754$ & $25.313 \pm 7.088$ & $<0.001^{\mathrm{c}}$ \\
\hline $\mathrm{R} 5\left(\mathrm{cmH}_{2} \mathrm{O} /(\mathrm{L} / \mathrm{s})\right)$ & $101.052 \pm 27.009$ & $216.334 \pm 67.657$ & $<0.001^{\mathrm{c}}$ \\
\hline $\mathrm{R} 20\left(\mathrm{cmH}_{2} \mathrm{O} /(\mathrm{L} / \mathrm{s})\right)$ & $96.620 \pm 27.588$ & $157.634 \pm 24.796$ & $<0.001^{\mathrm{a}}$ \\
\hline $\mathrm{R} 5-\mathrm{R} 20\left(\mathrm{cmH}_{2} \mathrm{O} /(\mathrm{L} / \mathrm{s})\right)$ & $10.208 \pm 7.351$ & $63.944 \pm 60.949$ & $<0.001^{\mathrm{c}}$ \\
\hline $\mathrm{X} 5\left(\mathrm{cmH}_{2} \mathrm{O} /(\mathrm{L} / \mathrm{s})\right)$ & $0.599 \pm 0.451$ & $4.190 \pm 2.570$ & $<0.001^{\mathrm{c}}$ \\
\hline
\end{tabular}

Data are presented as mean \pm SD; the values of WBC\#, neu\#, eos\#, neu\%, and eos\% were in blood. The values of FSTL1 were in plasma; BMI: body mass index; WBC\#: white blood cell count; neu\#: neutrophil count; eos\#: eosinophil count; neu\%: neutrophil percentage; eos\%: eosinophil percentage; $\mathrm{FEV}_{1}$ : forced expiratory volume in one second; $\mathrm{FEV}_{1} / \mathrm{FVC}$ : forced expiratory volume in one second/forced vital capacity; Zrs: respiratory system impedance; Fres: resonant frequency; R5: resistance at $5 \mathrm{~Hz}$; R20: resistance at $20 \mathrm{~Hz}$; X5: reactance at $5 \mathrm{~Hz}$; R5-R20: R5 was subtracted by R20; ${ }^{\mathrm{a}}$ two-tailed independent sample $t$-test; ${ }^{\mathrm{b}}$ Pearson chi-square test; ${ }^{\mathrm{c}}$ separate variance estimation $t$-test; ${ }^{\mathrm{d}}$ Mann-Whitney $U ;{ }^{\ddagger} n=21$.

Immunohistochemisty (IHC) was performed on $5 \mu \mathrm{m}$ thick paraffin-embedded sections of human lung samples. Slides were deparaffinized and pretreated with $1 \mathrm{mmol} / \mathrm{L}$ EDTA and heat-mediated antigen retrieval solution (Solarbio) in a microwave oven. The antigen was blocked in $20 \%$ normal fetal bovine serum (ZSGB, Beijing, China). Sections were incubated with rabbit anti-FSTL1 antibody (Abcam, Cambridge, UK) at $1: 100$ dilution, rabbit anticollagen I (Bioss, MA, USA) at 1:100 dilution, and mouse anti- $\alpha$-SMA (BOSTER, CA, USA) at $1: 100$ dilution. Sections were then incubated with peroxidase-conjugated goat anti-rabbit IgG (ZSGB, Beijing, China) at 1:500 dilution and peroxidase-conjugated goat anti-mouse IgG (ZSGB) at 1:500 dilution, followed by addition of streptavidinHRP conjugate and substrate-chromogen mixture. Finally, all slides were counterstained with hematoxylin.

Quantification of staining on histological images was performed using Image at $\times 400$ magnification. Images were corrected for white balance before color deconvolution. We selected the all the noncontiguous regions of interest (ROI) to calculate the integral optical density (IOD). By dividing IOD by the area, we can get the average optical density (AOD). For the expression of each kind of protein (FSTL1, $\alpha$-SMA, and collagen I), three sections of IHC were used to calculate the AOD per subject. Five random areas of each section were measured in each PSAM staining slides to determine RBM thickness and fibrosis levels. Results are the mean of three slides per patient in $\mu \mathrm{m}$.
2.6. Follow-Up of Asthmatic Patients. After one month, more than three quarters of our enrolled asthmatics returned to our clinic for follow-up, of whom 17 subjects were well controlled and did not suffer from any other respiratory complications, such as pneumonia. Four months after their initial visit, 10 subjects returned for follow-up. Blood samples were collected for routine blood, and spirometry was performed. Subjects received treatment (inhaled corticosteroids and long-acting $\beta$-agonist combination therapy and/or oral leukotriene receptor antagonists) for their asthma after their first visit.

2.7. Statistical Analysis. All data are presented as mean \pm standard deviation (SD). Data was first tested for normality using the Shapiro-Wilk test before analysis with the appropriate ANOVA test. Plasma levels of FSTL1 were logarithmically transformed $\left(\log _{10}\right)$ because of their skewed distribution. FSTL1 and other parameters were examined by Spearman rank correlation analysis. A value of $p<0.05$ was considered as statistically significant. All analyses were performed using SPSS (version 20) (IBM, NY, USA). The quantitative analysis of figures of IHC, PASM staining, and Sirius red staining was performed using ImageJ.

\section{Results}

3.1. Characteristics across Controls and Asthmatics. 32 patients with asthma completed our study, among whom 


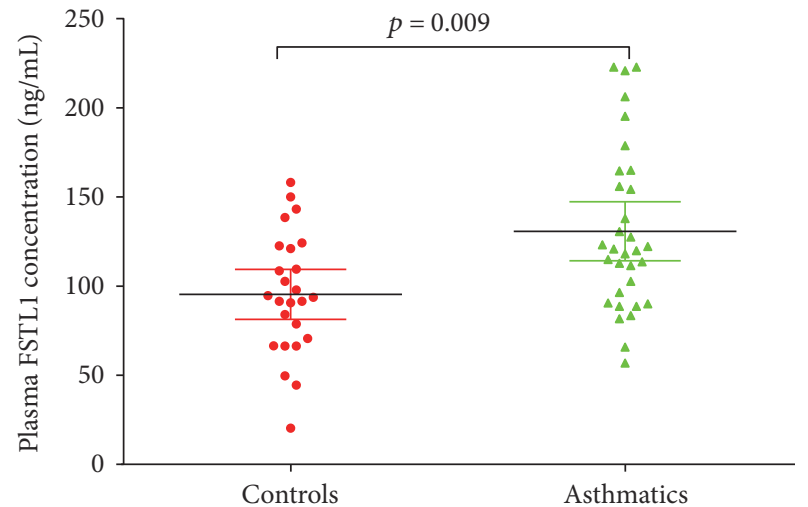

(a)

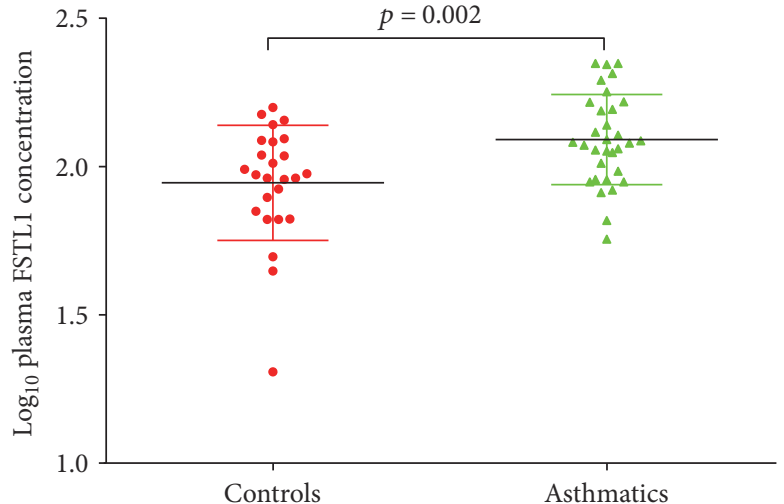

(b)

Figure 1: Plasma FSTL1 levels in controls and asthmatics. (a) Plasma FSTL1 levels in controls and asthmatics. (b) $\log _{10}$ FSTL1 levels in controls and asthmatics from plasma. Data is represented as mean \pm SD.

TABLE 2: Spearman rank correlation analysis to identify factors associated with $\log _{10}$ FSTL1 in asthmatics.

\begin{tabular}{lcc}
\hline & Correlation coefficient & $p$ value \\
\hline Age $($ years $)$ & -0.241 & 0.185 \\
$\mathrm{BMI}\left(\mathrm{kg} / \mathrm{m}^{2}\right)$ & 0.081 & 0.658 \\
eos\# $\left(\times 10^{9} / \mathrm{L}\right)$ & 0.309 & 0.086 \\
eos\% & 0.253 & 0.163 \\
$\mathrm{FEV}_{1}(\%$ predicted $)$ & -0.459 & $\mathbf{0 . 0 0 8}$ \\
$\mathrm{FEV}_{1} / \mathrm{FVC}$ & -0.351 & $\mathbf{0 . 0 4 9}$ \\
$\mathrm{Zrs}(\Omega)$ & 0.157 & 0.389 \\
Fres $(1 / \mathrm{s})$ & 0.351 & $\mathbf{0 . 0 4 9}$ \\
$\mathrm{R} 5\left(\mathrm{~cm} \mathrm{H}_{2} \mathrm{O} /(\mathrm{L} / \mathrm{s})\right)$ & 0.365 & $\mathbf{0 . 0 4 0}$ \\
$\mathrm{R} 20\left(\mathrm{cmH}_{2} \mathrm{O} /(\mathrm{L} / \mathrm{s})\right)$ & 0.247 & 0.173 \\
$\mathrm{R} 5-\mathrm{R} 20\left(\mathrm{cmH} \mathrm{H}_{2} \mathrm{O} /(\mathrm{L} / \mathrm{s})\right)$ & 0.263 & 0.147 \\
$\mathrm{X} 5\left(\mathrm{cmH}_{2} \mathrm{O} /(\mathrm{L} / \mathrm{s})\right)$ & 0.162 & 0.375 \\
\hline
\end{tabular}

BMI: body mass index; eos\#: eosinophil count; eos\%: eosinophil percentage; $\mathrm{FEV}_{1}$ : forced expiratory volume in one second; $\mathrm{FEV}_{1} / \mathrm{FVC}$ : forced expiratory volume in one second/forced vital capacity; Zrs: respiratory system impedance; Fres: resonant frequency; R5: resistance at $5 \mathrm{~Hz}$; R20: resistance at $20 \mathrm{~Hz}$; X5: reactance at $5 \mathrm{~Hz}$; R5-R20: R5 was subtracted by R20.

11 were male $(34.4 \%)$. Of the 25 controls, 10 were male (40.0\%). Characteristics of the patients are described in Table 1. There was no difference between the groups in age, gender, or BMI.

The concentration $(p=0.006)$ and percentage $(p=0.008)$ of eosinophils in the blood, respiratory system impedance (Zrs) $(p<0.001)$, resonant frequency (Fres) $(p<0.001)$, resistance at $5 \mathrm{~Hz}(\mathrm{R} 5)(p<0.001)$, resistance at $20 \mathrm{~Hz}$ (R20) $(p<0.001)$, R5 minus R20 (R5-R20) $(p<0.001)$, and reactance at $5 \mathrm{~Hz}(\mathrm{X} 5)(p<0.001)$ were all higher in the asthma group compared to the control group. Forced expiratory volume in one second $\left(\mathrm{FEV}_{1}\right)$ and $\mathrm{FEV}_{1} /$ forced vital capacity (FVC) were higher in controls than in asthmatics $(p<0.001$ and $p<0.001$, resp.) (Table 1$)$. Of note, plasma concentrations of FSTL1 in the asthma group (130.762 \pm $46.029 \mathrm{ng} / \mathrm{mL})$ were significantly elevated compared to controls $(95.408 \pm 33.938 \mathrm{ng} / \mathrm{mL})(p=0.009)$ (Figure 1).
3.2. FSTL1 and Lung Function and IOS. There was a positive correlation between $\log _{10}$ FSTL1 levels in plasma and Fres ( $\mathrm{rs}=0.351, p=0.049$ ) as well as R5 ( $\mathrm{rs}=0.365, p=0.040$ ) (Table 2 and Figure 2). Plasma FSTL1 levels were inversely related to $\mathrm{FEV}_{1}$ (rs $\left.=-0.459, p=0.008\right)$ and $\mathrm{FEV}_{1} / \mathrm{FVC}$ (rs $=-0.351, p=0.049$ ) (Table 2 and Figure 2).

3.3. FSTL1 Levels in BALF with Asthma. BALF was collected from 15 asthmatics when they underwent fibre optic bronchoscopy before sampling. FSTL1 levels in BALF positively correlated with plasma FSTL1 concentration ( $\mathrm{rs}=0.550$, $p=0.034$ ) (Figure 3).

3.4. Staining of the Bronchial Biopsies. At high magnification, H\&E stains of asthmatic sections showed that the epithelium was mostly intact but infiltrated by inflammatory cells (Figure 4(b)). Epithelial cells were also longer than normal in asthmatic airways (Figures 4(a) and 4(b)). In normal controls, the reticular basement membrane contained few inflammatory cells (Figure 4(a)). Sloughed cells found in control sections were partly the result of postmortem changes and pathological changes due to diseases other than asthma (Figure 4(a)).

IHC staining demonstrated higher FSTL1 expression in asthmatic sections than in controls $(p<0.001)$ (Figures 4(c), 4(d), and 4(e)). FSTL1 expression in control sections was mostly in the epithelia and nonspecific staining on the top of the ciliated cells (Figure 4(c)). However, in asthmatic sections, FSTL1 was expressed not only in mesenchymal cells but also in epithelia (Figure 4(d)).

Similarly, expression of $\alpha$-SMA and collagen I were higher in asthmatics than in nonasthmatic controls ( $p<0.001$ and $p<0.001$, resp.) (Figures 4(f), 4(g), 4(h), 4(i), $4(\mathrm{j})$, and $4(\mathrm{k}))$. IHC staining showed that $\alpha$-SMA was expressed in the vascular endothelial cells in controls. However, $\alpha$-SMA was highly expressed in both vascular endothelial cells and the submucosa in asthmatics, demonstrating increased smooth muscle mass in asthmatics (Figures 4(f) and $4(\mathrm{~g})$ ). In controls, mesenchymal cells expressed collagen 


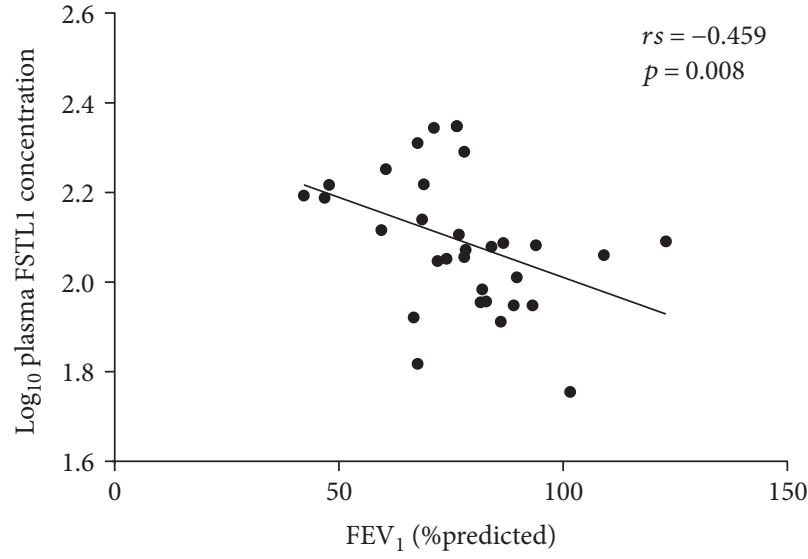

(a)

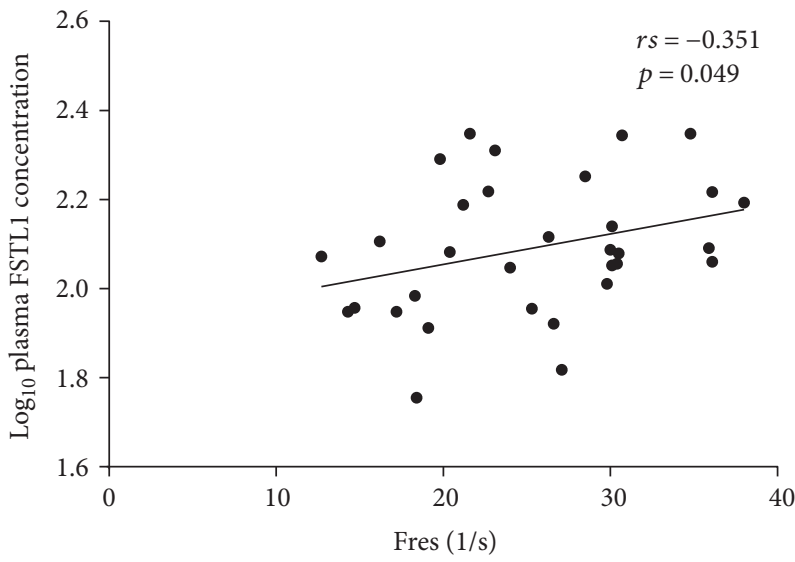

(c)

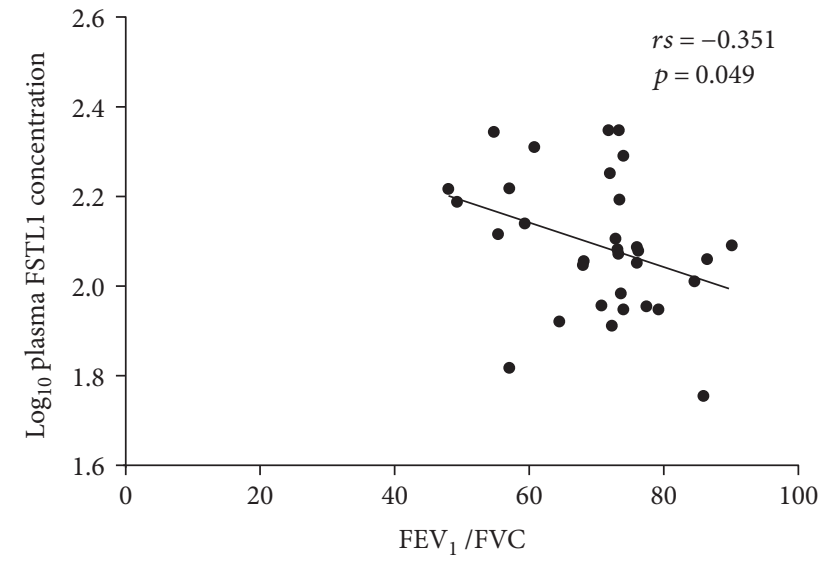

(b)

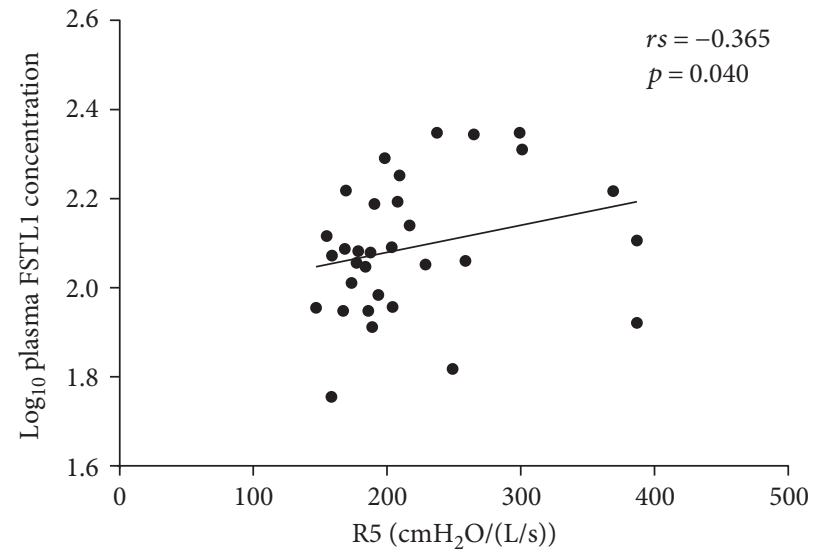

(d)

FIGURE 2: Correlation between plasma $\log _{10}$ FSTL1 levels and spirometric and IOS parameters. Correlations between plasma log ${ }_{10}$ FSTL1 levels and (a) $\mathrm{FEV}_{1}$ (\%predicted), (b) $\mathrm{FEV}_{1} / \mathrm{FVC}$, (c) Fres, and (d) R5. Correlations were determined by Spearman rank correlation analysis.

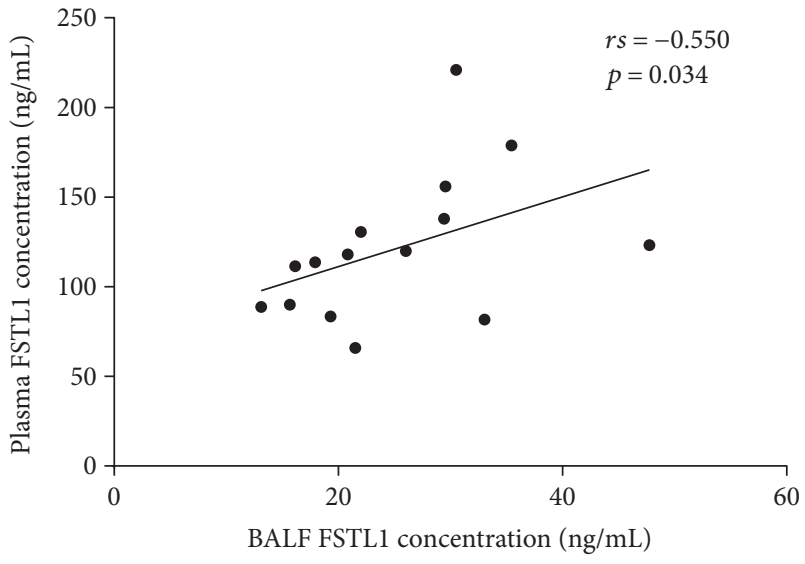

FIgURE 3: Correlation between FSTL1 levels in plasma and BALF. Correlation was determined by Spearman rank correlation analysis.

I (Figure 4(i)). However, in asthmatic sections, epithelial cells also stained positive for collagen I (Figure $4(\mathrm{j})$ ).

PASM staining of the airway basement membrane was greater in asthmatics compared to that in controls
(Figures $4(\mathrm{l})$ and $4(\mathrm{~m})$ ) and was determined quantitatively to be thicker in asthmatic sections $(p<0.001)$ (Figure $4(\mathrm{n})$ ).

Sirius red staining for fibrosis around the airways showed that there was increased collagen deposition around the airway in asthmatics compared with that in the controls (Figures 4(o), 4(p), and 4(q)).

3.5. Correlation between FSTL1 and Airway Remodeling. Bronchial biopsies of 15 asthmatics were collected, and Spearman rank correlation analysis demonstrated that plasma FSTL1 levels were correlated positively with the expression of $\alpha$-SMA in bronchial biopsies ( $\mathrm{rs}=0.554$, $p=0.032$ ) (Figure 5(a)) and fibrosis levels around the airways $(\mathrm{rs}=0.529, p=0.043)$ (Figure 5(c)). Furthermore, the levels of BALF FSTL1 correlated with the expression of $\alpha$-SMA ( $\mathrm{rs}=0.561, p=0.029$ ) (Figure 5(b)), fibrosis levels around the airways ( $\mathrm{rs}=0.779, p=0.001$ ) (Figure $5(\mathrm{~d})$ ), thickness of basement membrane ( $\mathrm{rs}=0.660, p=0.007)$ (Figure 5(f)), and collagen I ( $\mathrm{rs}=0.536, p=0.040$ ) (Figure $5(\mathrm{~h})$ ).

3.6. Changes before and after Treatment. Figure 6 shows the changes of observed values before treatment, one month after treatment, and four months after treatment. During the 


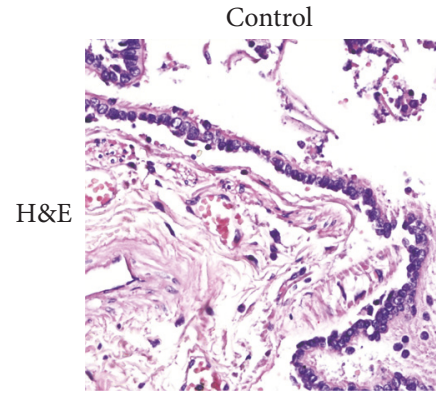

(a)

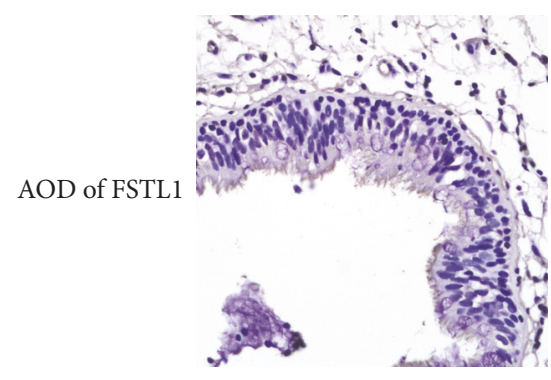

(c)



(f)

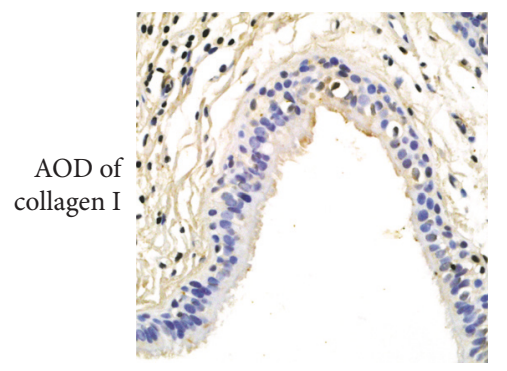

(i)

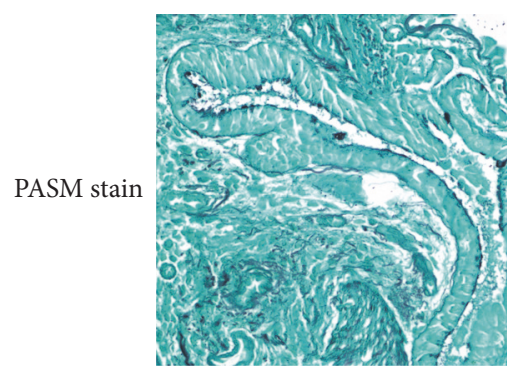

(1)

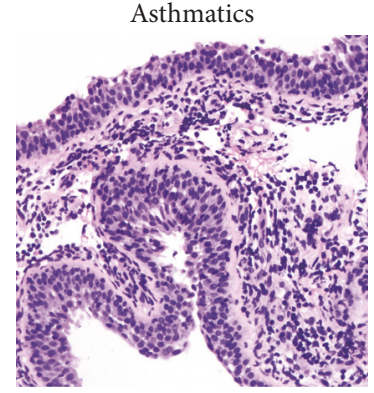

(b)

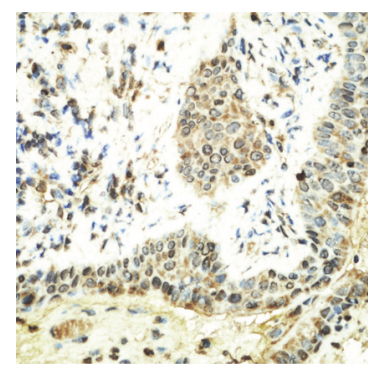

(d)

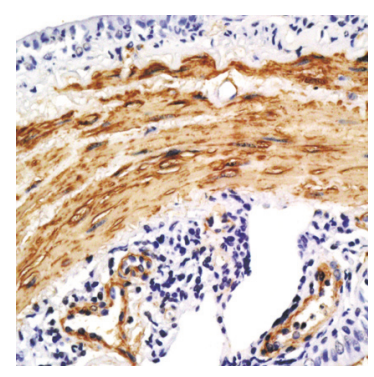

(g)

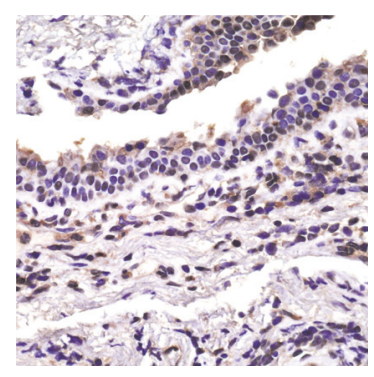

(j)

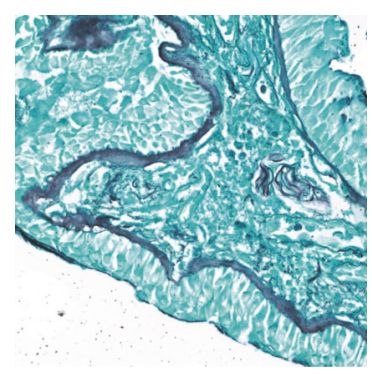

(m)

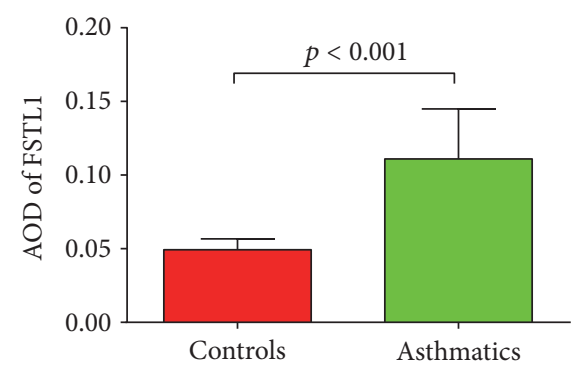

(e)

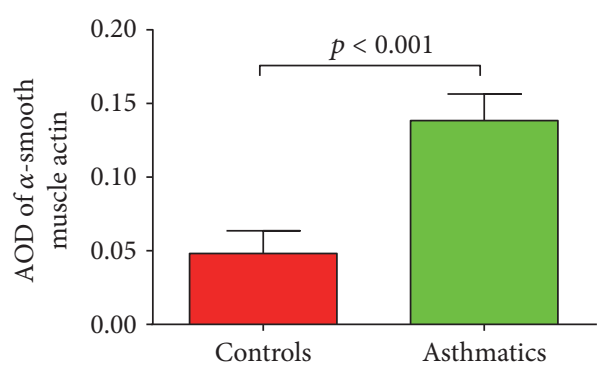

(h)



(k)



(n)

FIgURE 4: Continued. 


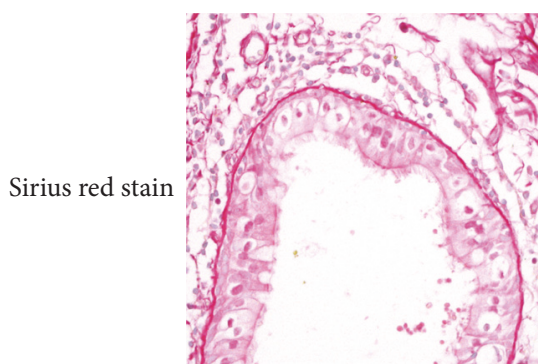

(o)

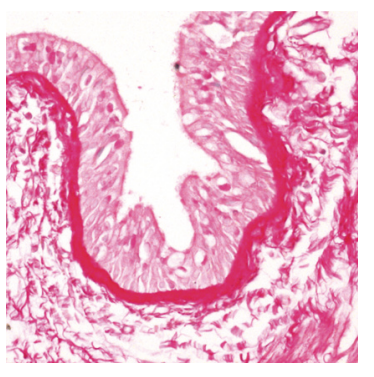

(p)

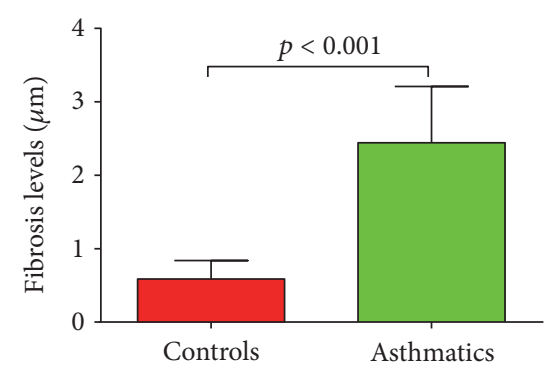

(q)

FIgURE 4: Staining of bronchial biopsies of both controls (postmortem samples) and asthmatics and quantitative analysis. H\&E stains for (a) controls and (b) asthmatics. Expression of FSTL1 by IHC in (c) controls and (d) asthmatics and (e) quantification of FSTL1 staining. Expression of $\alpha$-smooth muscle actin by IHC in (f) controls and (g) asthmatics and (h) quantification of $\alpha$-smooth muscle actin staining. Expression of collagen I by IHC in (i) controls and (j) asthmatics and (k) quantification of collagen I staining. Expression of basement membrane thickness by PASM staining in (l) controls and (m) asthmatics and (n) quantification of basement membrane staining. Expression of fibrosis levels by Sirius red staining in (o) controls and (p) asthmatics and (q) quantification of fibrosis levels. Original magnification $\times 400$. Data is represented as mean \pm SD.

period of follow-up, the concentration of FSTL1 in plasma decreased over time from pretreatment $(143.519 \pm 53.194 \mathrm{ng} /$ $\mathrm{mL})$ to one month after treatment $(28.447 \pm 10.325 \mathrm{ng} / \mathrm{mL})$ $(p<0.001)$ (Figures 6(c) and 6(d)). However, there were no changes between plasma FSTL1 levels between one-month follow-up $(28.447 \pm 10.325 \mathrm{ng} / \mathrm{mL})$ and four months $(28.023$ $\pm 12.891 \mathrm{ng} / \mathrm{mL})$ after treatment $(p=0.996)$ (Figures $6(\mathrm{c})$ and $6(\mathrm{~d})$ ). With regard to $\mathrm{FEV}_{1}$ and $\mathrm{FEV}_{1} / \mathrm{FVC}$, there was a general increasing trend; however, the changes were not statistically significant (Figures 6(a) and 6(b)).

\section{Discussion}

In this study, we demonstrated that the levels of FSTL1 in the plasma were elevated in asthmatics versus those in age- and gender-matched controls. For asthmatics, there was a correlation between plasma FSTL1 levels and $\mathrm{FEV}_{1}, \mathrm{FEV}_{1} / \mathrm{FVC}$, Fres, R5, and FSTL1 levels in BALF. IHC staining of human bronchial biopsies and quantitative analysis demonstrated that FSTL1 was expressed more highly in biopsies of asthmatics than in those of controls. Furthermore, plasma FSTL1 levels correlated with the expression of $\alpha$-SMA in bronchial biopsies and fibrosis levels around the airways. BALF FSTL1 concentrations were correlated with the expression of collagen I and $\alpha$-SMA, the thickness of reticular basement membrane, and fibrosis levels around the airways. We found that the concentration of plasma FSTL1 decreased significantly after 1 month of treatment with inhaled corticosteroids and long-acting $\beta$-agonists combination therapy and/ or oral leukotriene receptor antagonists.

Our study demonstrated that there was a relationship between FSTL1 levels in plasma and BALF, with concentrations being higher in plasma than those in BALF. We speculate that during the bronchoscopy procedure where BALF was collected, the routinely used saline diluted the FSTL1 concentration. The relationship between FSTL1 levels in plasma and the parameters of airway remodeling was weaker than the relationship between FSTL1 levels in BALF and parameters of airway remodeling, suggesting its importance in the remodeling process. Although we set strict inclusive and exclusive criteria, subjects enrolled in this study may have had some other underlying conditions, which could influence the concentration of FSTL1, especially plasma concentration. Despite this, FSTL1 levels in plasma did relate to airway remodeling of parameters to a certain degree since they were linked with expression of $\alpha$-SMA in bronchial biopsies and fibrosis levels around the airways, suggesting some utility of its use as a blood biomarker.

The increased expression of FSTL1 in biopsies was accompanied by more expression of $\alpha$-SMA collagen I and a thicker reticular basement membrane, which are all the hallmarks of airway remodeling in asthma [2, 30]. The thickened reticular basement membrane has been reported to be due to the deposition of collagens I and III, tenascin, fibronectin, and immunoglobulin (Ig), which can lead to subepithelial fibrosis [31]. The relationship between hallmarks of airway remodeling and FSTL1 levels in BALF demonstrated that FSTL1 levels could reflect the degree of reticular basement membrane thickening and smooth muscle mass increase. Our study demonstrated that plasma FSTL1 levels were correlated with fibrosis levels around airways and smooth muscle mass increase, but not the expression of the collagen I and basement membrane thickness. Considering that plasma FSTL1 levels fluctuate and decrease with asthma treatment and the specificity of BALF FSTL1 concentrations with remodeling parameters, we speculate that FSTL1 may play an important role in airway remodeling.

Moreover, after treatment for asthma, the levels of FSTL1 in plasma declined, but due to moral and ethical reasons, we were unable to assess airway remodeling with additional bronchoscopy procedures. Inhaled corticosteroids and/or oral leukotriene receptor antagonists could decrease the expression of FSTL1 in plasma directly or indirectly. Given our findings that plasma and BALF FSTL1 concentrations correlate, we speculate that with treatment, BALF FSTL1 levels would also be reduced with treatment. Whether airway remodeling in asthma is halted or slowed 


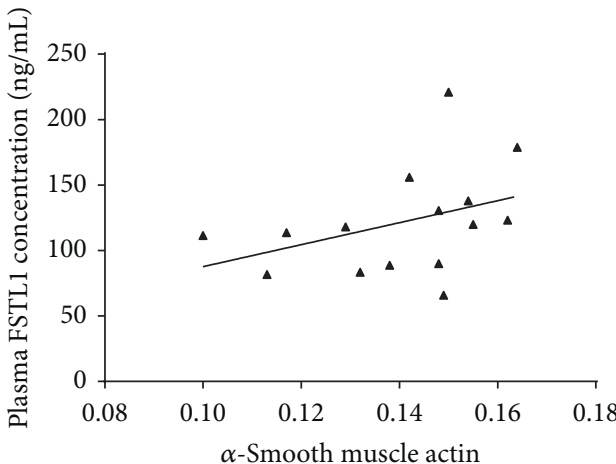

(a)

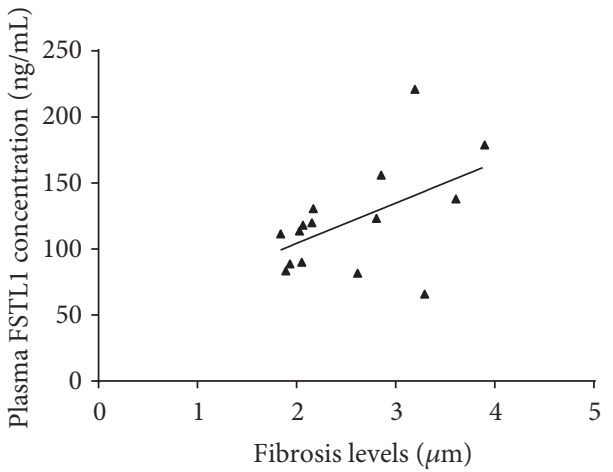

(c)

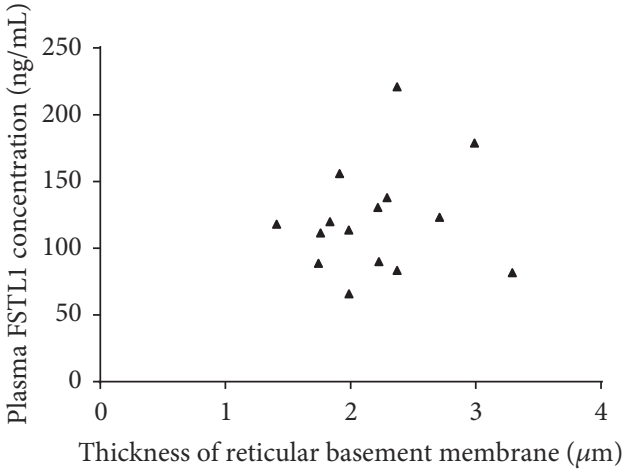

(e)

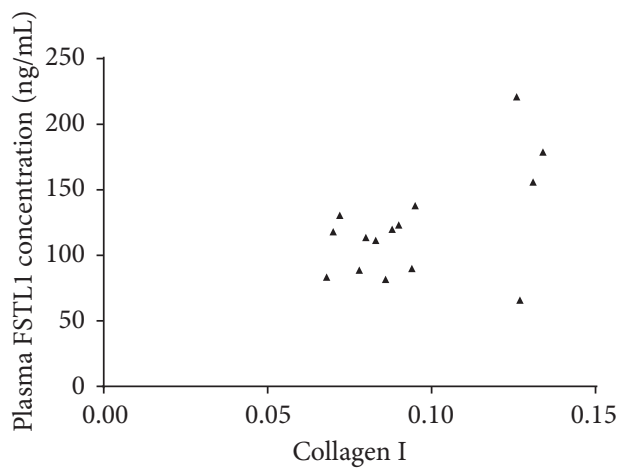

(g)

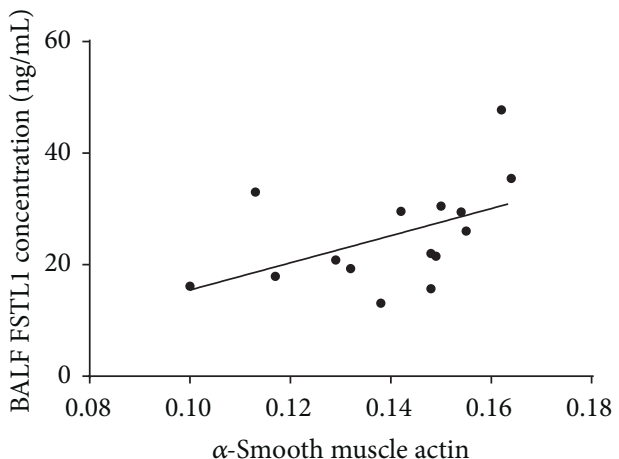

(b)

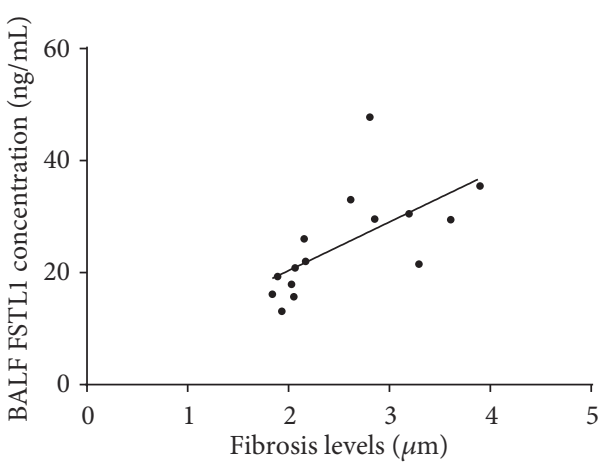

(d)

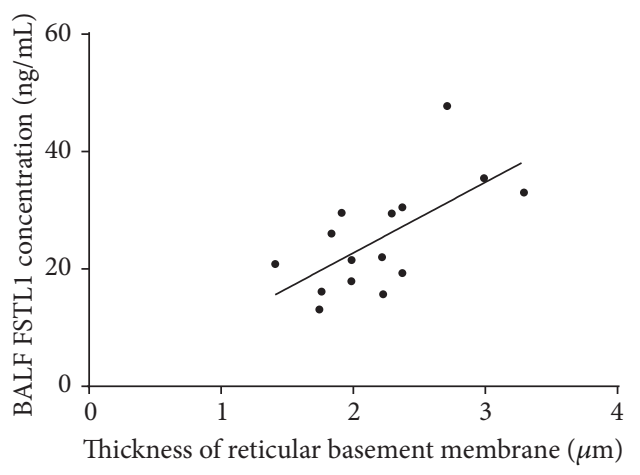

(f)



(h)

FIGURE 5: Relationships between FSTL1 levels in plasma and BALF and airway remodeling. Correlation between $\alpha$-smooth muscle actin and (a) plasma FSTL1 concentration and (b) BALF FSTL1 concentration. Correlation between fibrosis levels and (c) plasma FSTL1 concentration and (d) BALF FSTL1 concentration. Correlation between basement membrane thickness and (e) plasma FSTL1 concentration and (f) BALF FSTL1 concentration. Correlation between collagen I levels and (a) plasma FSTL1 concentration and (b) BALF FSTL1 concentration. Correlation was determined by Spearman rank correlation analysis. 


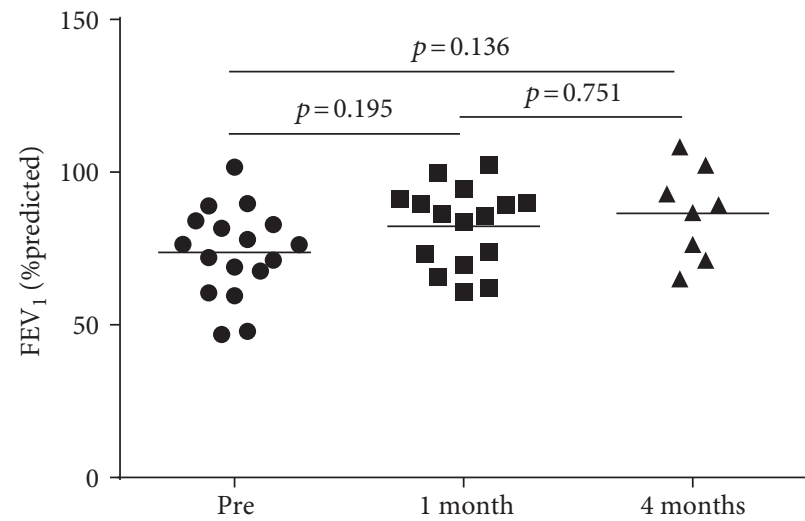

(a)

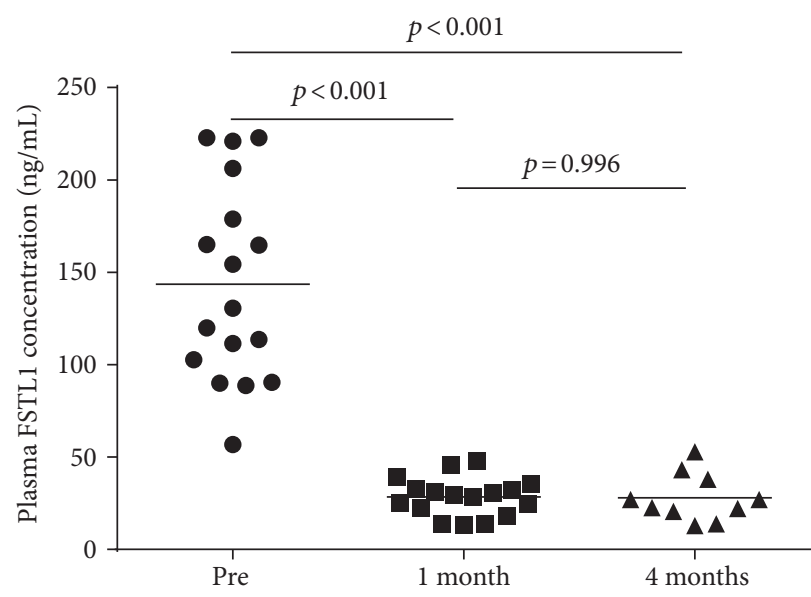

(c)

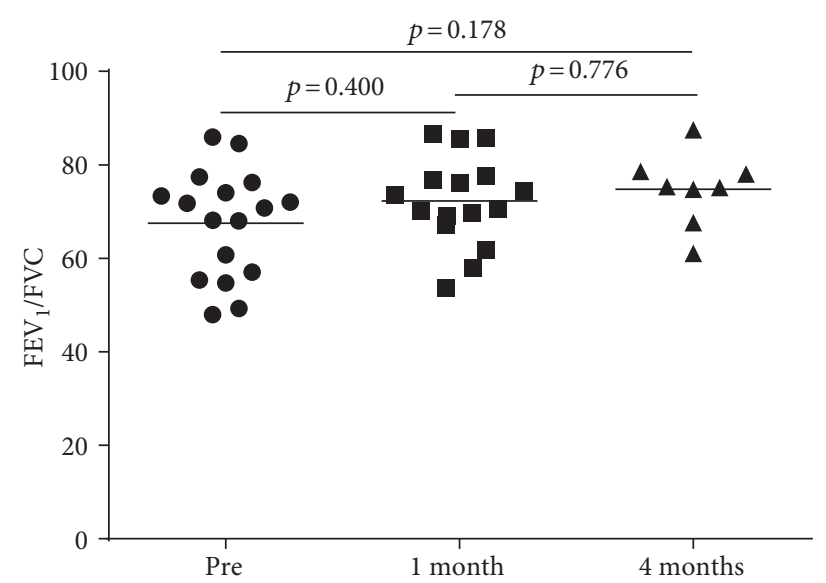

(b)

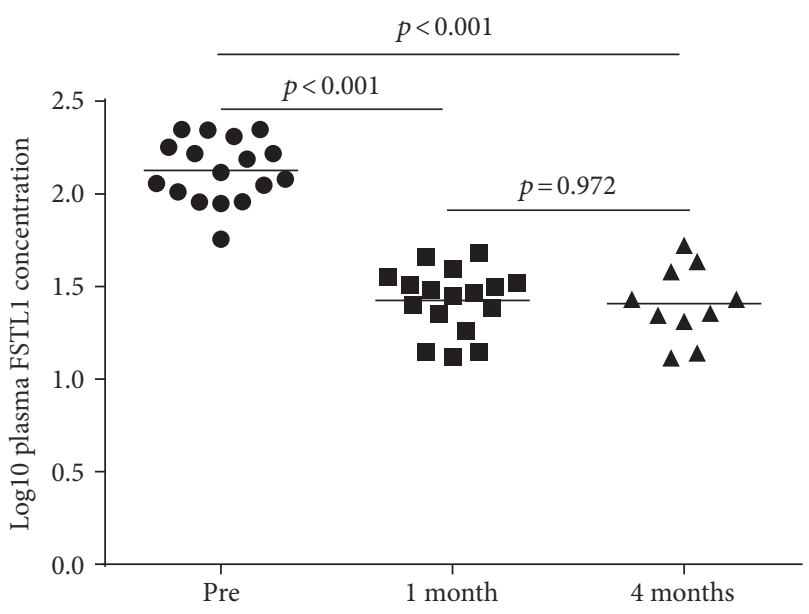

(d)

FIGURE 6: The changes of spirometry and FSTL1 levels before and after treatment. (a) FEV $_{1}$ (\%predicted) before treatment, 1 month posttreatment, and 4 months posttreatment. (b) $\mathrm{FEV}_{1} / \mathrm{FVC}$ before treatment, 1 month posttreatment, and 4 months posttreatment. (c) Plasma FSTL1 concentration before treatment, 1 month posttreatment, and 3 months posttreatment. (d) Log 10 plasma FSTL1 concentrations before treatment, 1 month posttreatment, and 3 months posttreatment. Multiple comparison was performed by Games-Howell test. Line indicates mean.

or if the decline of FSTL1 is linked with the improvement of pulmonary function, testing is still not clear and requires further investigation.

Since elevated levels of FSTL1 coexist with airway remodeling in asthma, we wonder whether FSTL1 participates in airway remodeling as a mediator or whether FSTL1 levels increase following airway remodeling as a kind of maladaption. For the time being, the mechanisms of airway remodeling in asthma are still unclear. Previous studies demonstrated that decreased levels of FSTL1 in heterozygous FSTL1 $^{+/-}$-deficient mice attenuated bleomycin-induced pulmonary fibrosis through a TGF- $\beta$-dependent pathway [27]. Miller et al. found that administration of FSTL1 to WT mice induces airway remodeling in a mouse model of asthma [28]. Investigation of molecular mechanisms was outside the scope of our study, but taking previous work in the literature into consideration, we believe that FSTL1 is likely to promote airway remodeling in asthma. However, the exact mechanisms by which this occurs still need to be elucidated.
In general, FSTL1 is secreted by nonhematopoietic cells such as cells of the mesenchymal lineage, such as fibroblasts [32]. Our study found that the airway epithelia also expressed FSTL1. However, the role of FSTL1 expression in epithelial cell is currently unknown and further studies are required to determine its role in airway remodeling.

Asthma is a heterogeneous disease, and according to Masoli et al., the global burden of asthma accounts for about $1 \%$ of disability-adjusted life years of all diseases around the world [33]. More effective therapies are needed and one of the hurdles to developing therapeutics is the lack of complete understanding of the mechanisms underlying the pathogenesis of this disease. Our findings here add new insights into the understanding of asthma and indicate a potential novel role of FSTL1 in asthma.

\section{Conclusion}

In summary, our study demonstrated that the levels of plasma FSTL1 were elevated in asthmatics and the levels of 
FSTL1 in plasma and BALF were positively correlated in people with asthma. There was an association between FSTL1 and airway remodeling in asthmatics without treatment suggesting that it may promote airway remodeling in people with asthma.

$\begin{array}{ll}\text { Abbreviations } \\ \text { FSTL1: } & \text { Follistatin-like protein } 1 \\ \text { BALF: } & \text { Bronchoalveolar lavage fluid } \\ \text { IOS: } & \text { Impulse oscillometry } \\ \text { ELISA: } & \text { Enzyme-linked immunosorbent assay } \\ \text { BMI: } & \text { Body mass index } \\ \text { WBC\#: } & \text { White blood cell count } \\ \text { Eos\#: } & \text { Eosinophil count } \\ \text { Eos\%: } & \text { Eosinophil percentage } \\ \text { neu\#: } & \text { Neutrophil count } \\ \text { neu\%: } & \text { Neutrophil percentage } \\ \text { FEV } 1: & \text { Forced expiratory volume in one second } \\ \text { FEV } / \text { FVC: } & \text { Forced expiratory volume in one second/forced } \\ & \text { vital capacity } \\ \text { Zrs: } & \text { Respiratory system impedance } \\ \text { Fres: } & \text { Resonant frequency } \\ \text { R5: } & \text { Resistance at } 5 \mathrm{~Hz} \\ \text { R20: } & \text { Resistance at } 20 \mathrm{~Hz} \\ \text { X5: } & \text { Reactance at } 5 \mathrm{~Hz} \\ \text { R5-R20: } & \text { R5 minus R20 } \\ \text { rs: } & \text { Spearman rank correlation coefficient. } \\ \end{array}$

\section{Conflicts of Interest}

The authors declare that there is no conflict of interest regarding the publication of this paper.

\section{Acknowledgments}

This work was supported by grants from the National Natural Science Foundation of China (Grant no. 81270072) and the Natural Science Funding Committee of Shandong province (Grant no. ZR2015PH022). Special thanks are given to David Broide and Francesca Tang who have contributed their time and talent to the review of this article.

\section{References}

[1] D. H. Broide, "Immunologic and inflammatory mechanisms that drive asthma progression to remodeling," The Journal of Allergy and Clinical Immunology, vol. 121, pp. 560-570, 2008, quiz 571-572.

[2] N. Hirota and J. G. Martin, "Mechanisms of airway remodeling," Chest, vol. 144, pp. 1026-1032, 2013.

[3] T. R. Bai, "Evidence for airway remodeling in chronic asthma," Current Opinion in Allergy and Clinical Immunology, vol. 10, pp. 82-86, 2010.

[4] L. James, T. R. Bai, T. Mauad et al., "Airway smooth muscle thickness in asthma is related to severity but not duration of asthma," The European Respiratory Journal, vol. 34, pp. 1040-1045, 2009.
[5] T. Doherty and D. Broide, "Cytokines and growth factors in airway remodeling in asthma," Current Opinion in Immunology, vol. 19, pp. 676-680, 2007.

[6] M. Shibanuma, J. Mashimo, A. Mita, T. Kuroki, and K. Nose, "Cloning from a mouse osteoblastic cell line of a set of transforming-growth-factor-beta 1-regulated genes, one of which seems to encode a follistatin-related polypeptide," European Journal of Biochemistry/FEBS, vol. 217, pp. 13-19, 1993.

[7] A. Zwijsen, H. Blockx, W. Van Arnhem et al., "Characterization of a rat C6 glioma-secreted follistatin-related protein (FRP): cloning and sequence of human homologue," European Journal of Biochemistry/FEBS, vol. 225, pp. 937-946, 1994.

[8] C. Kudo-Saito, T. Fuwa, K. Murakami, and Y. Kawakami, "Targeting FSTL1 prevents tumor bone metastasis and consequent immune dysfunction," Cancer Research, vol. 73, pp. 6185-6193, 2013.

[9] C. Kudo-Saito, "FSTL1 promotes bone metastasis by causing immune dysfunction," Oncoimmunology, vol. 2, 2013.

[10] K.-C. Li, F. Wang, Y.-Q. Zhong et al., "Reduction of follistatinlike 1 in primary afferent neurons contributes to neuropathic pain hypersensitivity," Cell Research, vol. 21, pp. 697-699, 2011.

[11] N. Fan, H. Sun, Y. Wang et al., "Follistatin-like 1: a potential mediator of inflammation in obesity," Mediators of Inflammation, vol. 2013, Article ID 752519, 12 pages, 2013.

[12] J. Wu, Y. Dong, X. Teng, M. Cheng, Z. Shen, and W. Chen, "Follistatin-like 1 attenuates differentiation and survival of erythroid cells through Smad2/3 signaling," Biochemical and Biophysical Research Communications, vol. 466, pp. 711-716, 2015.

[13] Y. Geng, Y. Dong, M. Yu et al., "Follistatin-like 1 (FSTL1) is a bone morphogenetic protein (BMP) 4 signaling antagonist in controlling mouse lung development," Proceedings of the National Academy of Sciences of the United States of America, vol. 108, pp. 7058-7063, 2011.

[14] M. Sylva, V. S. Li, A. A. Buffing et al., "The BMP antagonist follistatin-like 1 is required for skeletal and lung organogenesis," PLoS One, vol. 6, no. 8, article e22616, 2011.

[15] J. Xu, X. Qi, J. Gong et al., "Fstl1 antagonizes BMP signaling and regulates ureter development," PLoS One, vol. 7, no. 4, article e32554, 2012.

[16] Y. Yang, J. Liu, H. Mao, Y. A. Hu, Y. Yan, and C. Zhao, "The expression pattern of follistatin-like 1 in mouse central nervous system development," Gene Expression Patterns, vol. 9, pp. 532-540, 2009.

[17] K.-C. Li, F.-X. Zhang, C.-L. Li et al., "Follistatin-like 1 suppresses sensory afferent transmission by activating Na+,K+-ATPase," Neuron, vol. 69, pp. 974-987, 2011.

[18] Y. Chaly, A. D. Marinov, L. Oxburgh, D. S. Bushnell, and R. Hirsch, "FSTL1 promotes arthritis in mice by enhancing inflammatory cytokine/chemokine expression," Arthritis \& Rheumatology, vol. 64, pp. 1082-10888, 2012.

[19] Y. Chaly, H. C. Blair, S. M. Smith et al., "Follistatin-like protein 1 regulates chondrocyte proliferation and chondrogenic differentiation of mesenchymal stem cells," Annals of the Rheumatic Diseases, vol. 74, pp. 1467-1473, 2014.

[20] Y. Chaly, Y. Fu, A. Marinov et al., "Follistatin-like protein 1 enhances NLRP3 inflammasome-mediated IL- $1 \beta$ secretion from monocytes and macrophages," European Journal of Immunology, vol. 44, pp. 1467-1479, 2014. 
[21] S. Maruyama, K. Nakamuram, K. N. Papanicolaou et al., "Follistatin-like 1 promotes cardiac fibroblast activation and protects the heart from rupture," EMBO Molecular Medicine, vol. 8, pp. 987-1113, 2016.

[22] M. Shimano, N. Ouchi, K. Nakamura et al., "Cardiac myocyte follistatin-like 1 functions to attenuate hypertrophy following pressure overload," Proceedings of the National Academy of Sciences, vol. 108, pp. E899-E906, 2011.

[23] Y. Ogura, N. Ouchi, K. Ohashi et al., "Therapeutic impact of follistatin-like 1 on myocardial ischemic injury in preclinical models," Circulation, vol. 126, pp. 1728-1738, 2012.

[24] X. Li, Y. Fang, X. Li et al., "Apical secretion of FSTL1 in the respiratory epithelium for normal lung development," PLoS One, vol. 11, no. 6, article e0158385, 2016.

[25] M. Sylva, V. S. Li, A. A. Buffing et al., "The BMP antagonist follistatin-like 1 is required for skeletal and lung organogenesis," PLoS One, vol. 6, no. 8, article e22616, 2011.

[26] K. Bae, K. E. Park, J. Han, J. Kim, K. Kim, and K.-A. Yoon, "Mitotic cell death caused by follistatin-like 1 inhibition is associated with up-regulated Bim inactivated Erk1/2 in human lung cancer cells," Oncotarget, vol. 7, 2016.

[27] Y. Dong, Y. Geng, L. Li et al., "Blocking follistatin-like 1 attenuates bleomycin-induced pulmonary fibrosis in mice," The Journal of Experimental Medicine, vol. 212, pp. 235-252, 2015.

[28] M. Miller, A. Beppu, P. Rosenthal et al., "FSTL1 promotes asthmatic airway remodeling by inducing oncostatin," Journal of Immunology, vol. 195, pp. 3546-3556, 2015.

[29] M. R. Miller, J. A. Hankinson, V. Brusasco et al., "Standardisation of spirometry," The European Respiratory Journal, vol. 26, no. 2, pp. 319-338, 2005.

[30] P. G. Woodruff and J. V. Fahy, "Airway remodeling in asthma," Seminars in Respiratory and Critical Care Medicine, vol. 23, pp. 361-367, 2002.

[31] W. R. Roche, J. H. Williams, R. Beasley, and S. T. Holgate, "Subepithelial fibrosis in the bronchi of asthmatics," Lancet, vol. 333, pp. 520-524, 1989.

[32] Y. Chaly, B. Hostager, S. Smith, and R. Hirsch, "Follistatinlike protein 1 and its role in inflammation and inflammatory diseases," Immunologic Research, vol. 59, no. 1-3, pp. 266-272, 2014.

[33] M. Masoli, D. Fabian, S. Holt, and R. Beasley, "The global burden of asthma: executive summary of GINA dissemination committee report," Allergy, vol. 59, pp. 468-478, 2004. 


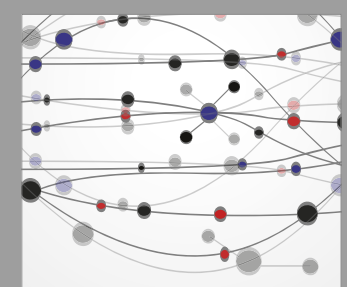

The Scientific World Journal
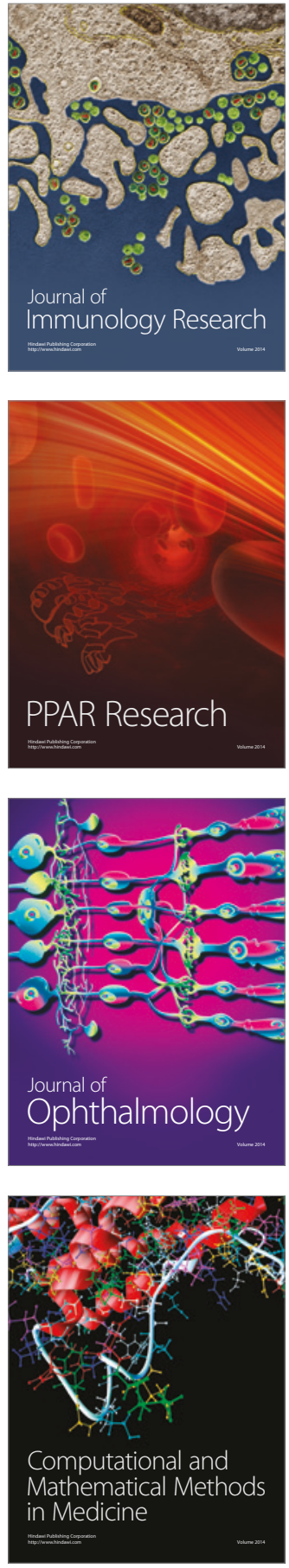



Gastroenterology Research and Practice
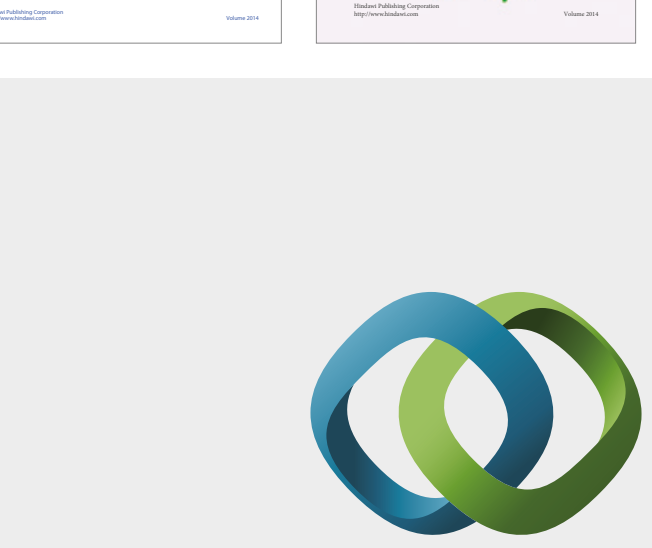

\section{Hindawi}

Submit your manuscripts at

https://www.hindawi.com



Disease Markers
